\title{
PRACTICE e ICPC-2 na abordagem de pacientes acometidos por cefaleia crônica diária
}

\author{
PRACTICE and ICPC-2 in the approach of patients affected by \\ chronic daily headache
}

\begin{abstract}
Rafael de Tasso Almada Picardi, Ariovaldo Alberto da ilva Júnior, Ruth Borges Dias, Enio Rodrigues da Silva, Paulo de Tasso Almada Picardi, Fidel Castro Meira, Vanessa Vilela Caires, Frederico Siqueira Araújo, Neson Morozini Júnior, Anna Carolina Gelini, Bianca Wilke Carvalho, Bruna Sobreira Britto, Camila Catizani Alvin, Camila Marcondes dos Santos, Carla Caroline Barreto Cunha, Carla Cassiana Souza Bueno, Francine Luiza Seganfredo, Jane Carolina Guimarães, Larissa Figueirêdo Carvalho, Laura Campos Egídio, Luiz Paulo Nunes Ferreira Tomaz, Marcelo Vassalo Visciani, Márcia Andrea Coutinho Mattos, Maria Rita de Souza Costa, Mariella Perraro Martins, Patrícia Bernardes Silva, Rômulo de Carvalho Quadros Barros, Thays Marchi

Picardi RTA, Silva AA, Jr, Dias RB, Silva ER, Picardi PTA, Meira FC, Caires W, Araújo FS, Morozini N Jr, Gelini AC, Carvalho BW, Britto BS, Alvin CC, Santos CM, Cunha CCB, Bueno CCS, Seganfredo FL, Guimarães JC, Carvalho LF, Egídio LC, Tomaz LPNF, Visciani MV, Mattos MAC, Costa MRS, Martins MP, Silva PB, Barros RCQ, Marchi T. Practice e ICPC-2 na abordagem de pacientes acometidos por cefaleia crônica diária. Headache Medicine. 2012;3(4):173-80
\end{abstract}

\section{RESUMO}

Introdução: A CCD caracteriza-se por quinze episódios dolorosos mensais nos últimos três meses consecutivos. $\mathrm{Na}$ gênese do quadro encontram-se fatores como comorbidades psiquiátricas, abuso de analgésicos e fragilidade sociofamiliar. Abordar tais aspectos constitui tarefa importante na sua condução. Objetivos: Descrever a utilização do PRACTICE e ICPC-2 na abordagem da CCD. Avaliar quantitativamente o resultado da codificação pelo ICPC-2. Metodologia: Pacientes consecutivos apresentando CCD foram entrevistados utilizando o PRACTICE. A ferramenta compõe-se de sete domínios que exploram a dinâmica familiar no enfrentamento do problema. As informações foram codificadas pela ICPC2, que permite a classificação das queixas da maneira enunciada, utilizando um sistema biaxial compreendendo sistemas orgânicos, psicológicos e sociais no primeiro eixo e as características do relato no segundo. Resultados: Foram abordados 14 pacientes, $86 \%$ mulheres e média de idade 42,7 anos. O diagnóstico mais frequente foi cefaleia por abuso de medicação, abrangendo $86 \%$ dos casos, com período de evolução médio da doença de 18,7 anos, e frequência semanal média de episódios dolorosos de 5,2 dias. Os códigos ICPC mais relevantes foram P01 (57,1\%); P03 (57,1\%); P18 $(85,7 \%)$; Z10 (50\%); Z20 (85,7\%). Conclusões: A amostra apresentou características compatíveis com a literatura. A codificação pela ICPC-2 indicou fatores reconhecidamente envolvidos na manutenção do quadro. $O$ presente estudo é o primeiro utilizando as ferramentas PRACTICE e ICPC-2 na CCD e nas cefaleias em geral. A codificação dos dados qualitativos obtidos pelo PRACTICE através da ICPC-2, possibilitando a análise quantitativa dos relatos, não foi utilizada em outros trabalhos pelo conhecimento dos autores até o momento.

Palavras-chave: Cefaleia; Cefaleia crônica diária; Questionários; Atenção primária à saúde

\section{ABSTRACT}

Chronic daily headache $(\mathrm{CDH})$ is characterized by 15 days with headache per month in the last three months. In its genesis, factors such as psychiatric comorbidities and painkillers abuse can be listed. It is important to address these factors. Objectives: To describe the use of PRACTICE and ICPC-2 in $\mathrm{CDH}$ and quantitatively evaluate the result of ICPC-2 coding. Methodology: Consecutive $\mathrm{CDH}$ patients were interviewed using PRACTICE. Seven domains, exploring the family dynamic facing the problem, compose this tool. The information obtained was coded using ICPC-2, which allows to classify the complaints in the manner they were spoken, using a biaxial system in which the organic, psychological and social systems are in the first axis and the account characteristics of are in the second one. Results: 14 patients were approached (86\% 
women, average age 42,7 years old). The most frequent diagnosis was migraine associated to medication overuse (86\%), with an illness average duration of 18,7 years, and weekly average frequency of painful episodes of 5,2 days. Conclusions: The sample characteristics were in accordance to what is found in the literature. Coding by ICPC-2 indicated known factors involved in clinical presentation maintenance. The present paper is the first using the PRACTICE tools and ICPC-2 in the approach of CDH and migraine. The authors are not aware of any previous paper describing the qualitative data coding obtained by PRATICE through ICPC-2.

Keywords: Headache; Chronic daily headache; Questionnaires; Primary health care

\section{INTRODUÇÃO}

A Cefaleia Crônica Diária (CCD) constitui síndrome caracterizada pela presença de cefaleia em pelo menos 15 dias por mês num período mínimo de três meses. ${ }^{(1)}$ Trata-se de uma síndrome e não de um diagnóstico etiológico, ${ }^{(2)}$ mas o termo CCD é usado de forma corrente nos centros especializados em cefaleia, tendo em vista que é muito comum a demanda por atendimento de pacientes que sofriam de cefaleias episódicas e que com o tempo passaram a apresentá-la de forma diária ou quase diária. ${ }^{(3)} \mathrm{O}$ conceito de CCD foi proposto por Silberstein et al. ${ }^{(4)}$ e abriga cefaleias primárias (as não relacionadas a alterações estruturais ou funcionais) e secundárias (as em que uma causa básica pode ser identificada), uma vez que o conceito expressa temporalidade e não etiopatogenia.(2) As principais afecções que evolvem clinicamente como CCD e são nosologicamente contempladas pela International Classification of Headache Disorders (ICHD-II)(5) são a migrânea crônica (MC; CID-10 G43.3), a cefaleia do tipo tensional crônica (CTTC; G44.2) e a cefaleia por abuso de medicação (CAM; G44.41).(1,2,5,6)

Diversos estudos populacionais estimaram a prevalência de CCD em cerca de $3 \%$ a $5 \%$ da população mundial em geral, ${ }^{(7-10)}$ dados corroborados em estudos conduzidos no Brasil. ${ }^{(1-14)}$ Tal prevalência gera impacto significativo em termos econômicos e de saúde pública. Estima-se que $9 \%$ das consultas em atenção primária ${ }^{(14)}$ e quase $30 \%$ das consultas a neurologistas ${ }^{(15)}$ resultem de queixa principal de cefaleia, enquanto a CCD é responsável por $80 \%$ ou mais de todos os pacientes atendidos em serviços especializados em cefaleia ${ }^{(3)}$ e por até $70 \%$ dos atendimentos por cefaleias em centros terciá- rios. ${ }^{(16)}$ A migrânea crônica, previamente conhecida por migrânea transformada na literatura, ${ }^{(1,2,3)}$ constitui a mais prevalente causa de CCD, atingindo cerca de $75 \%$ desses pacientes. ${ }^{(17)} \mathrm{A}$ cefaleia do tipo tensional atinge $8 \%$ de prevalência na $C C D,{ }^{(16)}$ e o abuso de medicação, que pode ocorrer em todos os tipos de CCD, pode chegar a $80 \%$ dos pacientes. ${ }^{(3,17)}$ Abuso de medicamentos sintomáticos caracteriza dificuldade no diagnóstico diferencial e tratamento da CCD, uma vez que se relaciona com a perda das características clínicas clássicas das cefaleias, ${ }^{(18)}$ não permitindo o diagnóstico diferencial antes de dois meses após sua interrupção, ${ }^{(19)}$ e também com a perda em efetividade do tratamento profilático e aumento da taxa de refratariedade. (20) $\bigcirc$ abuso de medicação analgésica é independentemente associada à piora nos escores de qualidade de vida (QV) dos pacientes. ${ }^{(21)}$ A prevalência de comorbidades psiquiátricas em pacientes acometidos por CCD e abusadores pode chegar a $87,5 \%$. ${ }^{(22)}$ De fato, ainda não se conhece o mecanismo exato pelo qual a cefaleia se cronifica, mas a inter-relação com o uso abusivo de analgésicos e as comorbidades psiquiátricas vem sendo bem estabelecida, conforme tem mostrado diversos estudos realizados tanto em centros de atendimento especializado quanto na própria comunidade. ${ }^{23,24)}$

O elevado impacto da CCD na QV é bem documentado tanto através de estudos com questionários gerais de QV quanto em questionários específicos para QV em cefaliatria. ${ }^{(25)} A$ presença de estresse atinge até 90\% dos pacientes com CCD, sendo que pacientes nessa situação pontuam significativamente menos nos questionários de $Q V$, sugerindo que estresse e baixa qualidade de vida estão relacionados tanto ao desenvolvimento quanto à manutenção da CCD. ${ }^{(26)}$

O tratamento da CCD deve ser individualizado e integral, baseando-se em medidas farmacológicas e não farmacológicas, ${ }^{(27)}$ compreendendo medidas gerais comportamentais, como mudança no estilo de vida, atividade física aeróbica regular e aprendizado da adaptação aos estressores, e medidas farmacológicas para analgesia e profilaxia. No caso da CAM, é imperativo o abandono do abuso de analgésicos para o sucesso do tratamento, interrupção dificultada pela frequência de sintomas de abstinência, que podem ocorrem em até 98\% dos casos, ${ }^{(27)}$ e pela ocorrência de piora clínica da cefaleia durante um período de 03 a 06 meses após a cessação do abuso, ${ }^{(28)}$ o que dificulta a aderência do paciente. Porém cerca de $29,7 \%$ dos pacientes não retornam a um padrão episódico de dor, causa frequente de reincidência 
no abuso e falha terapêutica. ${ }^{(29)} \bigcirc$ prognóstico da CCD ainda é motivo de controvérsia, mas sabe-se que longos períodos de abuso de medicações analgésicas relacionam-se a piores prognósticos. ${ }^{(27)}$

Processos psíquicos, sociais e familiares estão envolvidos na transformação e manutenção do quadro e não podem ser negligenciados. ${ }^{(2)}$ São comuns na prática clínica os relatos de pacientes envolverem questões psicossociais e sociofamiliares, seja identificando gatilhos para as crises, justificando o abuso medicamentoso ou evidenciando impacto da dor nos sistemas sociofamiliares que muitas vezes retroalimenta e favorece a cronificação, aspectos reconhecidos por profissionais da área como desafiadores na sua abordagem, mas pouco estudados em pesquisas quantitativas e qualitativas adequadas. $\bigcirc$ núcleo familiar cada vez mais é valorizado e privilegiado como foco das políticas sociais no Brasil, (30) haja vista a existência de programas de destaque mundial como Bolsa Família e a Estratégia de Saúde da Família (ESF), transformando-se na unidade básica de atenção social e à saúde. ${ }^{(31)}$ Tal fato reflete-se bem no manejo de doenças crônicas de alta prevalência como hipertensão arterial sistêmica (HAS) e diabetes mellitus no âmbito da ESF, onde diversas ferramentas específicas de abordagem familiar são comumente utilizadas com bons resultados, sendo consideradas um acréscimo ao tratamento convencional. ${ }^{(32)} \bigcirc$ manejo da CCD, no entanto, encontra-se ainda distante dessa realidade. Pode-se dizer que as cefaleias como um todo carecem da valorização na saúde pública que seu impacto econômico e social bem documentado deveria garantir.

Buscando suprir essa lacuna, ampliar o cuidado, melhorar a escuta aos pacientes e captar as nuances sociais e familiares ao mesmo tempo em que se produzam dados científicos quantitativos, comparáveis e consistentes, o presente estudo buscou ferramentas da medicina de família e comunidade capazes de auxiliar essa tarefa.

\section{FERRAMENTA PRACTICE}

O acróstico PRACTICE (Presenting problem; Roles and structure; Affect; Communication; Time in life cycle; Illness in family; Coping with stress; Ecology) consiste num instrumento de entrevista semiestruturada utilizado para o entendimento da dinâmica familiar no enfrentamento de problemas. Pode ser utilizado para organizar informações adquiridas da família em intervenções individuais ou grupais, sempre de forma objetiva e focada no problema em questão. Serve como guia na investigação do problema e pode-se realizá-lo mais como conversa que como entrevista, sem seguir rigidamente sua ordem, mas em geral inicia-se a aplicação do instrumento definindose o problema apresentado, no caso, a cefaleia crônica diária de um dos membros. Esse primeiro momento pouco difere da queixa principal de uma avaliação formal.

No segundo passo, Roles and structure, identificamse as regras e estrutura familiar, ou seja, explicita-se o papel de cada membro do agrupamento familiar no sistema e propõe-se a reflexão acerca das mudanças de papéis ocorridas durante as crises de cefaleia ou ao longo do período de cronificação da doença. Exemplos de estruturas familiares possíveis são a família nuclear, formada por familiares consanguíneos do paciente em foco, possuindo geralmente um núcleo de casal com seus filhos; as famílias monoparentais, formadas por um dos pais biológicos e um ou mais filhos; a família reconstituída, composta por membros de uma família que sofreu uma ruptura e passou a exibir uma nova configuração.

Affect investiga o afeto entre os membros da família diante do problema apresentado, os sentimentos de cada membro, identificando pontos de apoio emocional tanto quanto de tensão. A seguir busca-se compreender o padrão de comunicação familiar, tanto verbal quanto não verbal, em Communication. A definição do momento no ciclo de vida da família ocorre através da observação do entrevistador, ou seja, sem a necessidade de exteriorizar a questão, e auxilia a identificar as tensões mais comumente apresentadas ao longo de cada uma das fases evolutivas do grupamento familiar. Exemplos de Time in life cycle possíveis são as famílias recém-constituídas, as famílias com filhos adolescentes e as famílias chamadas de "ninho vazio", onde o processo de desligamento dos filhos adultos é iminente ou recente. Em Illness in family investigam-se também outras doenças importantes na dinâmica familiar, presentes e passadas. A penúltima etapa explora as estratégias de enfrentamento de situações estressoras dos indivíduos. Há indivíduos, por exemplo, que assumem posturas proativas diante dos problemas, enquanto outros desestabilizam-se ou deprimem, sem direcionar seus esforços para a solução do problema.

Por fim, em Ecology produz-se o genograma/ecomapa que permite ampliar e visualizar graficamente as relações familiares, entre as diversas gerações (sugerese que o genograma estude no mínimo três gerações da família) e a rede de apoio social, econômico e cultural, bem como identificar situações de fragilidade social. A 
ferramenta utiliza símbolos padronizados para construir a representação gráfica da dinâmica familiar capturada nos itens anteriores, auxiliando a família a perceber fragilidades na relação entre seus membros e com a comunidade e demonstrando o impacto de eventos ocorridos com um dos membros sobre os demais. Por resgatar essas informações, o genograma/ecomapa pode ensejar oportunidade de reflexões acerca do problema; facilitar o planejamento de intervenções; enfatizar barreiras e padrões de comunicação e explorar aspectos emocionais. Mais que uma ferramenta de coleta de dados, é parte do processo terapêutico. ${ }^{(31)} O$ Anexo 01 apresenta o PRACTICE de um sujeito da pesquisa plenamente preenchido. Tal abordagem é comumente utilizada na atenção primária brasileira e na ESF.

\section{FERRAMENTA ICPC-2}

Ao longo das décadas de 60 e 70 diversos pesquisadores buscaram desenvolver uma classificação que atendesse às necessidades da atenção primária, onde grande parte das vezes o médico lida com problemas e não com diagnósticos. Após a conferência de Alma Ata em 1978 esses esforços foram potencializados pelo interesse da Organização Mundial de Saúde (OMS), um processo que culminou em 1987 com a publicação da International Classification of Primary Care (ICPC). Uma revisão em 1998 possibilitou a comparabilidade de seus códigos com a Classificação Internacional de Doenças (CID-10). ${ }^{(33)}$ A International Classification of Primary Care, $2^{\text {nd }}$ edition (ICPC-2) $)^{(34)}$ consiste nessa revisão da ICPC e atua de maneira complementar à CID-10 nos serviços de atenção primária à saúde. ${ }^{(35)}$ Sua estrutura permite a classificação das queixas do paciente da maneira como são enunciadas, através de um sistema biaxial que compreende os sistemas orgânicos, psicológicos e sociais no primeiro eixo enquanto o segundo eixo contempla as características do relato. ${ }^{(35)} \bigcirc$ primeiro eixo é sempre composto por uma letra e o segundo sempre por um número. A ICPC-2 é composta por dezessete capítulos que podem corresponder ao primeiro eixo do código, a saber, geral e inespecífico (A); sangue, órgãos hematopoiéticos e linfáticos $(B)$; digestivo $(D)$; olho $(F)$; ouvido $(H)$; circulatório $(\mathrm{K})$; musculoesquelético $(L)$; neurológico $(\mathrm{N})$; psicológico $(P)$; respiratório $(R)$; pele $(P)$; endocrinometabólico e nutricional $(T)$; urológico $(U)$; gravidez e planejamento familiar (W); aparelho genital feminino $(X)$; aparelho genital masculino $(Y)$ e problemas sociais (Z). Estudos nacionais demonstram a confiabilidade interobservador desse instrumento, sugerindo um bom desempenho do mesmo na codificação dos dados de inquéritos de saúde compostos de questões abertas. ${ }^{(35)}$

$O$ presente artigo tem como objetivo descrever a utilização da codificação do PRACTICE através do ICPC2 na abordagem da CCD num ambulatório de referência secundária em neurologia.

\section{MÉTODOS}

Trata-se de estudo transversal realizado em centro de atendimento secundário em neurologia.

O CEASC da Unifenas-BH constitui a referência para atendimento neurológico das Unidades Básicas de Saúde (UBSs) da Região Norte de Belo Horizonte. A cidade de Belo Horizonte, com população de aproximadamente 2,4 milhões de habitantes em 2010, localiza-se no sudeste brasileiro e está dividida em nove regiões administrativas, cabendo à Regional Norte a responsabilidade por cerca de 200 mil habitantes, dos quais 11 mil famílias são beneficiários do Programa Bolsa Família, que repassa recursos a famílias com renda per capita de até R\$140,00.(36) A Regional Norte conta com 19 UBSs responsáveis pela atenção primária à saúde da população que referenciam casos de maior complexidade e que demandam atendimento especializado ao Centro de Especialidades Médicas Norte (CEM-Norte), que através de parceria com a Unifenas-BH funciona nas dependências do CEASC. O CEASC constitui unidade de ensino, contando com a participação de acadêmicos de medicina, enfermagem e nutrição, entre outros, no atendimento à população e aliando rigor acadêmico e científico aos princípios da humanização no atendimento à saúde presentes no Sistema Único de Saúde (SUS) do Brasil. Dados de estudo conduzido nessa unidade informam que a demanda por atendimento no ambulatório de neurologia por queixas principais de cefaleia é de $30,7 \%$, ${ }^{(37)}$ dificultando por limitações de tempo e número de profissionais a abordagem e intervenção nos aspectos psicossociais dos pacientes acometidos por CCD, em suma, o atendimento integral a esses pacientes.

Os participantes foram selecionados por médicos neurologistas no ambulatório de neurologia do Centro de Ensino e Atenção à Saúde da Comunidade Edson Antônio Vellano (CEASC) durante as consultas neurológicas. Ao longo do primeiro semestre de 2010, 14 pacientes consecutivos diagnosticados por neurologistas através do ICHD-II (International Classification of Headache Disorders - $2^{\text {nd }}$ Edition) $)^{(5)}$ e apresentando CCD 
foram entrevistados por acadêmicos de medicina utilizando a ferramenta PRACTICE.

Os pacientes foram convidados à participação na pesquisa através da assinatura de Termo de Consentimento Livre e Esclarecido (TCLE) previamente aprovado pelo Comitê de Ética em Pesquisa da Unifenas. As informações colhidas foram então codificadas utilizandose o ICPC-2.

Os resultados foram utilizados na discussão dos casos e na elaboração de estratégias de intervenção. O projeto foi previamente aprovado pelo Comitê de Ética em Pesquisa da Unifenas.

\section{RESULTADOS}

A amostra foi composta por 14 pacientes, $86 \%$ do sexo feminino. Quanto à idade, a amostra apresentou média de 42,71 anos, com mediana 38 e DP 12,06. A escolaridade, avaliada em anos frequentados sem levar em consideração o nível do ensino, apresentou média de 10,21 anos, mediana 11 e DP 3,78.

Quanto às características cefaliátricas da amostra, encontramos $86 \%$ de cefaleia por uso excessivo de medicação sintomática, $7 \%$ de migrânea crônica com aura e $7 \%$ de migrânea crônica sem aura. Em média os pacientes relataram um período de evolução diária ou quase diária da doença de 18,71 anos, com mediana 12,5 e DP 18,68. A frequência semanal de episódios dolorosos foi de 5,21 dias por semana em média, com mediana de 6 e DP 2,04.

A codificação do PRACTICE pelo ICPC-2 encontrou no domínio Presenting problem relatos mais frequentemente pertencentes ao capítulo psicológico do ICPC-2, como P01 (sensação de ansiedade/nervosismo/tensão) em 57,1\% dos casos; P03 (sensação de depressão) em 57,1\%; P04 (sentir/comportar de forma irritável/zangada) em 35,7\%; P06 (perturbação de sono) 14,3\%; P18 (abuso de medicação) em 85,7\% dos casos.

Os domínios Roles and structure; Affect; Communication e Ecology foram agrupados por abordarem aspectos sociofamiliares. Encontramos nesses domínios uma queixa psicológica frequente, P04 em $21,4 \%$. As demais queixas pertencem ao domínio social do ICPC-2, como ZO1 (pobreza/problemas econômicos) em 35,7\% dos casos; Z03 (problemas de habitação/ vizinhança) 35,7\%; Z05 (problemas com condições de trabalho) 14,3\%; Z06 (problema de desemprego) 14,3\%; Z10 (problema relacionado ao sistema de saúde) 50\%; Z1 1 (problema relacionado a estar doente)
14,3\%; Z12 (problema relacional com parceiro) 42,8\%; Z20 (problema relacional com familiares) $85,7 \%$; Z23 (perda/falecimento familiar) 28,6\%; Z25 (ato ou acontecimento violento) 21,4\%; Z24 (problema relacional com amigos) $21,4 \%$. O domínio Time in life cycle não foi codificado pelo ICPC-2. O domínio Illness in family encontrou relato de história familiar de N01 (cefaleia) em $50 \%$ dos casos.

A Figura 1 representa um exemplo de elaboração de PRACTICE e posterior codificação pelo ICPC-2 coletado na pesquisa.

\section{$\overline{\text { DISCUSSÃO }}$}

A principal limitação do estudo relaciona-se à exiguidade da amostra, apesar de termos encontrado resultados condizentes com a literatura de cefaleias. $\bigcirc$ achado de $86 \%$ da amostra pertencer ao sexo feminino encontra eco no estudo de Radat e cols.(38), em que as mulheres representavam $88,2 \%$ da população estudada em centro terciário. Na série clássica de Mathew e cols. ${ }^{(3)}$ a média de idade nos pacientes com migrânea transformada foi $41 \pm 12$ anos e a duração da migrânea episódica até a transformação foi de $16 \pm 11$ anos. Os participantes do presente estudo apresentaram idade média de 42,71 anos, com mediana 38 e DP 12,06 e relataram um período de evolução diária ou quase diária da doença de 18,71 anos, com mediana 12,5 e DP 18,68 .

Quanto às características cefaliátricas da amostra, o diagnóstico de CUEM em $86 \%$ é bem próximo da série de Mathew et al. que encontrou prevalência de abuso de medicações analgésicas em $87,2 \%$ dos casos. ${ }^{(3)} \bigcirc$ abuso, quando presente, é a maior causa de cronificação da dor $^{(4)}$ e relaciona-se à fisiopatologia da transformação da migrânea episódica em migrânea crônica possivelmente devido à diminuição do limiar de dor nos pacientes abusadores e ao prejudicar o efeito protetor das medicações profiláticas. ${ }^{(20)}$ Os pacientes abusadores também apresentam maior prevalência de comorbidades psiquiátricas. ${ }^{(22)}$

A ferramenta PRACTICE, ao ampliar o foco do relato para o espectro sociofamiliar, propicia ao paciente e ao médico a reflexão acerca de fatores relacionados à cronificação da cefaleia pouco abordados em consultas formais. A Figura 1 mostra um exemplo de preenchimento do PRACTICE e também um caso típico do esquema de cronificação da cefaleia após eventos estressores e seu impacto na dinâmica familiar da paciente. 


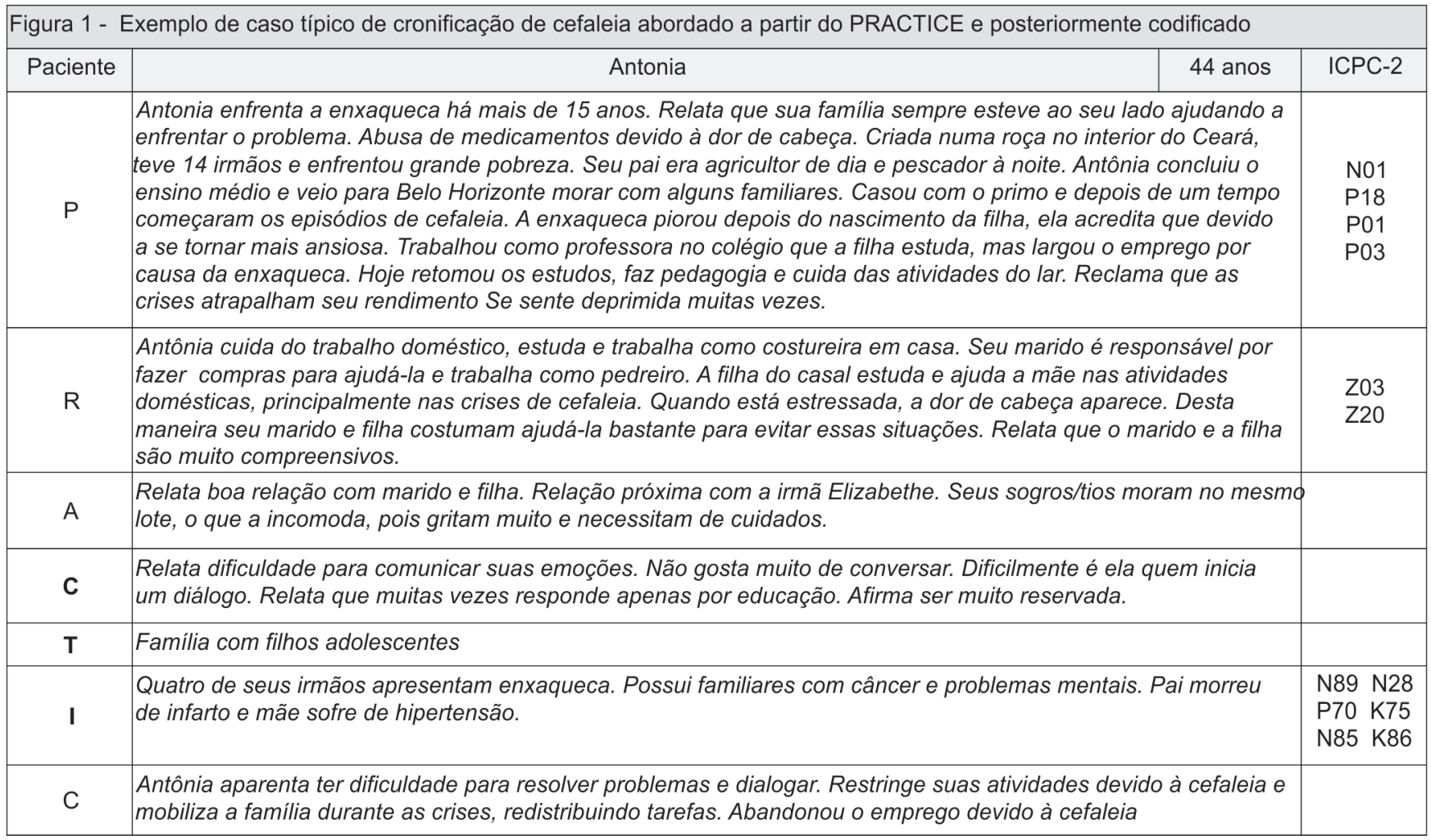

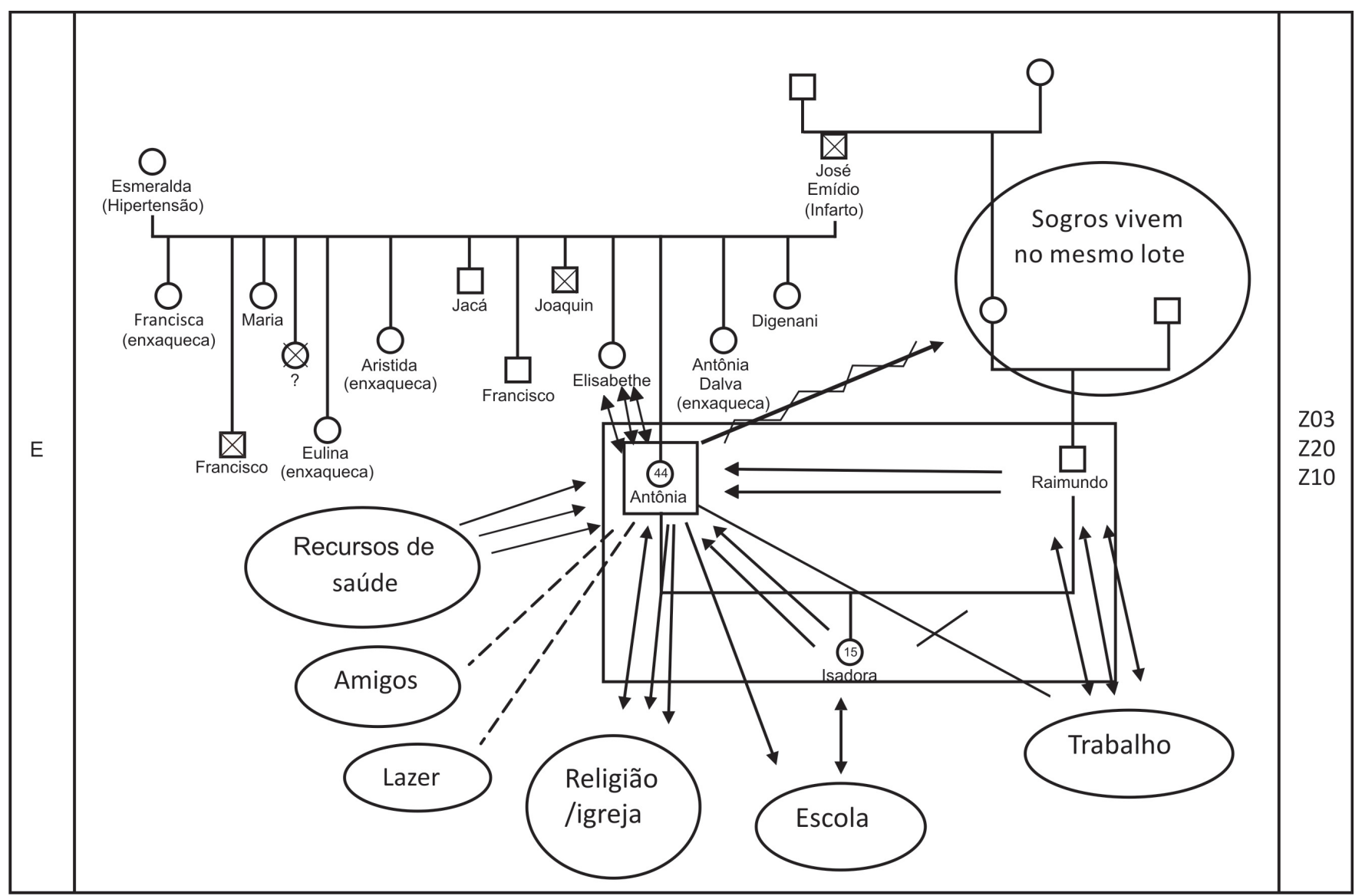


A codificação através do ICPC-2 encontrou alta prevalência de queixas reconhecidas por favorecer a cronificação. Os dados da codificação não são plenamente comparáveis com a literatura, onde os trabalhos buscam diagnósticos e não queixas, mas propiciam várias reflexões interessantes. Estudos encontraram transtornos ansiosos em $30 \%$ em pacientes migranosos ${ }^{(39)}$ e $57,1 \%$ em pacientes com CCD, (22) enquanto outros encontraram RR de 5,3 para transtorno de ansiedade generalizada, ${ }^{(40)}$ enquanto a queixa PO1 (sensação de ansiedade/ nervosismo/tensão) foi encontrada em $57,1 \%$ da amostra. A prevalência de transtornos depressivos na CCD pode chegar a 45,7\%; ; $^{(22)}$ encontramos o código P03 (sensação de depressão) em 57,1\% dos casos. Micieli et al. relacionaram a transformação da migrânea episódica em crônica com distúrbios do sono em 19\% dos casos; ${ }^{(41)}$ P06 (perturbação de sono) foi queixa em 14,3\% da amostra. Esses dados sugerem que seria interessante estudar o valor preditivo positivo das queixas psiquiátricas utilizando o ICPC-2.

Nos domínios PRACTICE relacionados a aspectos sócio-familiares, pode-se identificar códigos ICPC como Z20 (problema relacional com familiares) em $85,7 \%$ dos casos e Z05 (problemas com condições de trabalho) em $14,3 \%$, enquanto Sandrini et al. encontraram situações de estresse familiar em $21,3 \%$ e situações de estresse no trabalho em $16 \%$, sugerindo que o ICPC-2 possa ser mais sensível na identificação de atritos familiares. A literatura aponta que fatores existenciais traumáticos podem estar relacionados à cronificação na CCD em 11,9\% dos casos; ${ }^{(41)}$ segundo nossa amostra o ambiente sociofamiliar em 21,4\% dos pacientes se relaciona a Z25 (ato ou acontecimento violento).

O código Z10 (problema relacionado ao sistema de saúde) foi identificado em $50 \%$ dos casos. O fato levanta a problemática da elevada demanda desses pacientes aos serviços de saúde, fato muito comentado pelos profissionais e que carece de dados objetivos. $O$ código Z03 (problema relacionado a habitação/vizinhança) encontrado em 35,7\% dos casos pode ser explicado pela condição comum na região estudada de erigirem-se várias residências no mesmo lote, levando à convivência com extensos grupamentos familiares.

\section{CONCLUSÃO}

A amostra apresentou características compatíveis com a literatura. A codificação do ICPC-2 indicou fatores reconhecidamente envolvidos na manutenção do quadro.
O presente estudo é o primeiro utilizando as ferramentas PRACTICE e ICPC-2 na CCD e nas cefaleias em geral e a codificação dos dados qualitativos obtidos pelo PRACTICE através da ICPC-2, possibilitando a análise quantitativa dos relatos, não foi utilizada em outros trabalhos pelo que temos conhecimento até o momento. Os autores não encontraram trabalhos semelhantes na literatura nacional e internacional. Sua relevância clínica relaciona-se à possibilidade de ampliar o acolhimento e cuidado ao paciente através de ferramentas capazes de perceber o indivíduo como parte de um sistema biopsicossocial. A produção de dados quantitativos acerca de fenômenos sociofamiliares nessa população possibilita a materialização objetiva de percepções clínicas praticamente consensuais entre os cefaliatras que encontram pouco espaço em pesquisas científicas em parte devido a pouca familiaridade da maioria dos pesquisadores da área com pesquisas qualitativas. Sua relevância na saúde pública se dá através do desbravamento da utilização na CCD de instrumentos já amplamente utilizados para outras doenças crônicas no âmbito da ESF. Isso representa uma movimentação que pode com o tempo contribuir para corrigir a pouca atenção dada à CCD na ESF a despeito de sua elevada incidência na população em geral e do bem documentado impacto econômico e na qualidade de vida.

\section{REFERÊNCIAS}

1. Silberstein SD. Chronic daily headache. J Am Osteopath Assoc. 2005; 105(Suppl 2):23S-29.

2. Jevoux CC, Moreira Filho PF, Souza JA. Cefaléia crônica diária primária: características clínicas. Arq Neuropsiquiatr. 1998; 56(1):64-8.

3. Mathew NT, Reuveni U, Perez F. Transformed or evolutive migraine. Headache. 1987;27(2):102-6. Comment in: Headache. 2008; 48(5):728; discussion 729.

4. Silberstein SD, Lipton RB, Solomon S, Mathew NT. Classification of daily and near-daily headaches: proposed revisions to the IHS criteria. Headache 1994;34(1): 1-7.

5. Society. HCSotlH. The International Classification of Headache Disorders. 2nd Edition. Cephalalgia. 2004 24(Suppl 1):1149.

6. Bigal ME, Tepper SJ, Sheftell FD, Rapoport AM, Lipton RB. Chronic daily headache: correlation between the 2004 and the 1988 International Headache Society diagnostic criteria. Headache. 2004;44(7):684-91.

7. Rasmussen BK, Jensen R, Schroll M, Olesen J. Epidemiology of headache in a general population - a prevalence study. J Clin Epidemiol. 1991;44(1 1):1147-57.

8. Rasmussen BK. Epidemiology of headache. Cephalalgia. 1995; 15(1):45-68. 
9. Sandrini G, Manzoni GC, Zanferrari C, Nappi G. An epidemiological approach to the nosography of chronic daily headache. Cephalalgia. 1993;13(Suppl. 12):72-7.

10. Evers S, Suhr B, Baver B, Grotemeyer KH, Hussted IW. A retrospective long-term analysis of the epidemiology and features of drug-induced headaches. J Neurol. 1999;246(9): 802-9.

1 1. Silva AA, Jr, Krymchantowski AV, Moreira Filho PF, Vasconcelos LPB, Gomez RS, Teixeira AL. Prevalence of headache in the entire population of a small city in Brazil. Headache. 2009;49(6):895-9.

12. Queiroz LP, Barea LM, Blank N. An epidemiological study of headache in Florianopolis, Brazil. Cephalalgia. 2006;26 (2):122-7.

13. Queiroz LP, Peres MF, Kowacs F, Piovesan EJ, Ciciarelli MC, Souza JA, et al. Chronic daily headache in Brazil: a nationwide population-based study. Cephalalgia. 2008;28(12):1264-9.

14. Bigal ME, Bordini CA, Speciali JG. Etiology and distribution of headaches in two Brazilian primary care units. Headache. 2000; 40(3):241-7.

15. Ferri-de-Barros JE, Nitrini R. Que pacientes atende um neurologista? Alicerce de um currículo em neurologia. Arq Neuropsiquiatr. 1996;54:637-44.

16. Krymchantowski AV. Primary headache diagnosis among chronic daily headache patients. Arq Neuropsiquiatr. 2003; $61(2-B): 364-7$

17. Mathew NT, Stubits E, Nigam MP. Transformation of episodic migraine into daily headache: analysis of factors. Headache. $1982 ; 22(2): 66-8$.

18. Diener HC, Limmroth V. Medication overuse headache: a world wide problem. Lancet Neurol. 2004;3(8):475-83.

19. Krymchantowski AV, Barbosa JSS, Lorenzatto W, Cheim C, Adriano M. Características clínicas da migrânea transformada. Arq Neuropsiquiatr. 1999;57(4):990-3.

20. Weiller C, May A, Limmroth V, Jüptner M, Kaube H, Schayck RV, et al. Brain stem activation in spontaneous human migraine attacks. Nat Med. 1995; 1 (7):658-60.

21. Lipton RB, Hamelsky SW, Kolodner KB, Steiner TJ, Stewart WF. Migraine, quality of life and depression. A population- based case control study. Neurology 2000;55(5):629-35

22. Costa EAC. Comorbidades psiquiátricas na migrânea com e sem abuso de medicações analgésicas [dissertação]. Belo Horizonte: Universidade Federal de Minas Gerais; 2007.

23. Silva AA, Jr, Faleiros BE, Santos TM, Gómez RS, Teixeira AL. Relative frequency of headache types: a longitudinal study in the tertiary care. Arq Neuropsiquiatr. 2010;68(6):878-81.

24. Silva AA, Jr, Costa EAC, Gomes JB, Leite FM, Gomez RS, Vasconcelos LP, et al. Chronic headache and comorbibities: a two-phase, population-based, crosssectional study. Headache. 2010; 50(8):1306-12.

25. Zukerman E, Guendler VZ, Mercante JPP, Peres MFP. Cefaléia e qualidade de vida. Einstein. $2004 ; 2$ (Supl 1):73-5.

26. Galego JCB, Moraes AM, Cordeiro JA, Tognola WA. Chronic daily headache: stress and impact on the quality of life. Arq Neuropsiquiatr. 2007;65(4-B): $1126-9$.

27. Volcy-Gómez M. Cefalea crónica diaria: II. Tratamiento. Rev Neurol. 2005;40(3): 180-7.

28. Oliveira MF, Speciali JG. Cefaléia crônica diária: conceitos e tratamentos. Medicina, Ribeirão Preto. 2002;35:455-63.
29. Bigal M, Rapoport A, Sheftell F, Tepper S, Lipton R. Transformed migraine and medication overuse in a tertiary headache centerclinical characteristics and treatment outcomes. Cephalalgia. 2004; 24(6):483-90.

30. Itaboraí NR. A proteção social da família brasileira contemporânea: reflexões sobre a dimensão simbólica das políticas públicas. [site da Internet] 2005 [acessado em 2011 ago 19]: 18. Disponível em: http://www.abep. nepo.unicamp.br/ docs/anais/outros/FamPolPublicas/Nathalieltaborai.pdf

31. Moimaz SAS, Fadel CB, Yarid SD, Diniz DG. Family Health: the challenge of a collective attention. Cien Saude Colet. 201 1;16 Suppl 1:965-72. [Article in Portuguese].

32. Zanetti ML, Biagg MV, Santos MA, Peres DS, Teixeira CRS. O cuidado à pessoa diabética e as repercussões na família. Rev Bras Enferm. 2008;61 (2):186-92.

33. Gusso GDF. Diagnóstico de demanda em Florianópolis utilizando a Classificação Internacional de Atenção Primária: 2a edição (CIAP-2) [tese]. São Paulo: Universidade de São Paulo; 2009.

34. Comissão de Classificações da Organização Mundial de Ordens Nacionais, Academias e Associações Acadêmicas de Clínicos Gerais/ Médicos de Família (WONCA). Classificação internacional de cuidados primários. 2a ed. Oxford: Oxford University Press;1999.

35. Sampaio MMA, Coeli CM, Miranda NN, Faerstein E, Werneck GL, Chor D, et al. Confiabilidade interobservador da classificação internacional de cuidados primários. Rev Saúde Pública. 2008;42(3):536-4 1 .

36. Prefeitura Municipal de Belo Horizonte [homepage]. Belo Horizonte, MG: Prefeitura Municipal de Belo Horizonte; c2009 [atualizado em 2011 ; acessado em 19 de out. de 2011]. [4 telas]. Disponível em: http://portalpbh.pbh.gov.br/pbh/ecp/ comunidade.do? app = regionalnorte

37. Rodrigues JM, Caires W, Fontoura KBC, Silva TCS, Goulart SF, Teixeira AL, et al. Prevalence of headaches in individuals refered from the primary to the secondary care. Headache Medicine. No prelo 2011

38. Radat F, Sakh D, Lutz G, El Amrani M, Ferreri M, Bousser MG. Psychiatric comorbidity is related to headache induced by chronic substance use in migraineurs. Headache. 1999; 39(7):477 -80.

39. Breslau N, Davis GC, Andreski P. Migraine, psychiatric disorders, and suicide attempts: an epidemiologic study of young adults. Psychiatry Res. 1991; 37(1):11-23.

40. Merikangas KR, Angst J, Isler H. Migraine and psychopathology. Results of the Zurich cohort study of young adults. Arch Gen Psychiatry. 1990;47(9): 849-53.

41. Micieli G, Piazza D, Sinforiani E, Cavallini A, Trucco M, Gabellini $S$, et al. Antimigraine drugs in the management of daily chronic headaches: clinical profiles of responsive patients. Cephalalgia 1985;5(Suppl 2): 219-24.

Correspondência

Rafael de Tasso Almada Picardi Rua Flórida, 40, Apt 01 - Carmo 30310710 - Belo Horizonte, MG

Recebido: 07/12/2012

Aceito: 20/12/2012 